\title{
Control of the persistent currents in two interacting quantum rings through the Coulomb interaction and interring tunneling
}

\author{
L. K. Castelano, ${ }^{1}$ G.-Q. Hai, ${ }^{1, *}$ B. Partoens, ${ }^{2}$ and F. M. Peeters ${ }^{2}$ \\ ${ }^{1}$ Instituto de Física de São Carlos, Universidade de São Paulo, 13560-970 São Carlos, SP, Brazil \\ ${ }^{2}$ Department of Physics, University of Antwerp, Groenenborgerlaan 171, B-2020 Antwerp, Belgium
}

(Received 22 September 2008; published 19 November 2008)

\begin{abstract}
The persistent current in two vertically coupled quantum rings containing few electrons is studied. We find that the Coulomb interaction between the rings in the absence of tunneling affects the persistent current in each ring and the ground-state configurations. Quantum tunneling between the rings alters significantly the ground state and the persistent current in the system.
\end{abstract}

DOI: 10.1103/PhysRevB.78.195315

PACS number(s): 73.21.La, 05.30.Fk, 73.23.Ra, 85.35.Be

\section{INTRODUCTION}

Mesoscopic metallic rings and nanoscopic quantum rings (QRs) are known for the Aharonov-Bohm effect and its persistent current ${ }^{1}$ where the phase rigidity of the electron wave leads to quantum oscillations in the current. Recently, oscillatory persistent current was detected in self-assembled InAs/ GaAs semiconductor QRs having a single electron. ${ }^{2}$ Experimentally there have been a few approaches to fabricate coupled rings and little effort has been done on the system of vertically coupled QRs. ${ }^{3}$ Due to the ring geometry, these coupled ring complexes open a new route for measurement of quantum interference effects and for novel many-body states. ${ }^{4-7}$

Two vertically coupled quantum rings (CQRs) form a new type of artificial molecule. The interring distance and the tunneling strength together with the ring radius are new tunable parameters providing more degrees of freedoms to modulate and to control the electronic structure and the persistent current of these ring-shaped artificial molecules. In the present work, within the current spin-density functional theory (CSDFT), ${ }^{8,9}$ we study the persistent currents in the $\mathrm{CQRs}$ of few electrons. The two coupled rings exhibit new molecular many-body states. The tunability of the interring distance and the interring tunneling strength leads to a very rich variety of many-electron ground states and persistent current spectra. The persistent current and magnetization in a single ring have been studied extensively where the external magnetic field is the only parameter to alter the current at fixed ring radius. Here, we show that the persistent current in the CQRs depends on the interring distance and the tunneling strength between the two rings. Tunneling between the rings permits the exchange interaction between them inducing new quantum states that alter significantly the persistent current. This opens new ways to control the persistent current in quantum rings.

\section{THEORETICAL MODEL}

We first study the interring Coulomb interaction effects on the persistent current in two interacting QRs without tunneling. We model the QRs by a displaced parabolic potential $V(r)=\frac{1}{2} m^{*} \omega_{0}^{2}\left(r-r_{0}\right)^{2}$ in the $x y$ plane, where $\mathbf{r}=(x, y)=(r, \theta)$, $\omega_{0}$ is the confinement frequency, and $r_{0}$ is the radius of the ring. The two stacked identical rings are separated by a distance $d$ in the $z$ direction. They interact with each other through the Coulomb potential. A homogeneous magnetic field $\mathbf{B}=B \mathbf{e}_{z}$ is applied perpendicularly to the $x y$ plane, which is described by the vector potential $\mathbf{A}=\mathrm{Bre}_{\theta} / 2$ taken in the symmetric gauge. The Kohn-Sham orbitals $\psi_{j n m \sigma}(\mathbf{r})$ $=\exp (-i m \theta) \phi_{n m \sigma}(r) Z_{j}(z)$ are used to express the density and ground-state energy, where $\sigma=\uparrow$ or $\downarrow$ is the $z$ component of the electron spin and $\omega_{c}=e B / m^{*} c$ is the cyclotron frequency. The total density in the rings is $\rho(\mathbf{r})=\Sigma_{\sigma} \Sigma_{n, m}^{N_{\sigma}}\left|\phi_{n m \sigma}(\mathbf{r})\right|^{2}$. In the $z$ direction this density is approximated by a $\delta$ function in each ring. The interring Coulomb potential is given by

$$
V_{H}^{\text {inter }}(r)=\int d \mathbf{r}^{\prime} \frac{e^{2} \rho\left(r^{\prime}\right) / 2}{\varepsilon\left|\mathbf{r}-\mathbf{r}^{\prime}+\mathbf{d}\right|},
$$

with the interring distance $d=|\mathbf{d}|$. The exchange-correlation potential is considered within the local-density approximation.

In the CSDFT all the quantities are functionals depending on the spin-up $\left(\rho^{\uparrow}\right)$ and spin-down $\left(\rho^{\downarrow}\right)$ densities and the vorticity $\mathcal{V}(r)=\frac{m^{*} c}{e} \nabla \times \mathbf{j}_{p}(r) / \rho(r)$. Therefore the exchangecorrelation scalar and vector potential can also be written as a function of these quantities. ${ }^{8,10}$ The paramagnetic current density $\mathbf{j}_{p}(r)$ can be explicitly written as

$$
\mathbf{j}_{p}(r)=-\frac{\hbar}{m^{*} r} \mathbf{e}_{\theta} \sum_{\sigma} \sum_{n, m}^{N_{\sigma}} m\left|\phi_{n m \sigma}(r)\right|^{2} .
$$

The persistent current density, which is a measurable quantity, is given by $\mathbf{j}(r)=\mathbf{j}_{p}(r)+\left(e / m^{*} c\right) \rho(r) \mathbf{A}(r)$, where the second part corresponds to the diamagnetic current density. The persistent current is obtained by $I=\int j(r) d r=I_{p}+I_{d}$. We confirm that the magnetization in both the single and coupled QRs is proportional to the persistent current.

When interring quantum tunneling is permitted, the electronic states in different rings are coupled and the CQRs form an artificial molecule. There are bonding and antibonding states with an energy splitting $\Delta_{\text {SAS }}$ between the two levels. Tunneling occurs through a potential barrier between the rings in the $z$ direction $V(z)$ which is modeled by two coupled symmetric GaAs quantum wells of width $120 \AA$ and a barrier of $250 \mathrm{meV}$ height between them. Within this model, the energy splitting is given by $\Delta_{\mathrm{SAS}}$ 
$=22.86 \exp \left[-22.42 d\left(r_{0}\right)\right] \mathrm{meV}$ as a function of the interring separation. ${ }^{4}$ For the CQRs with interring tunneling, each ground-state (GS) can be labeled by three quantum numbers $\left(S_{z}, M_{z}, I_{z}\right)$, where the total spin $S_{z}$, total angular momentum $M_{z}$, and the isospin quantum number $I_{z}$.

\section{NUMERICAL RESULTS}

In the calculations, we consider GaAs quantum rings with a ring radius $r_{0}=2 a_{0}$ and a confinement energy $\hbar \omega_{0}$ $=5 \mathrm{meV}$, where $a_{0}=\sqrt{\hbar} / \mathrm{m}^{*} \omega_{0}$. The interring Coulomb interaction effects are studied by varying the distance $d$ between the two rings. The ground state (GS) of a single ring can be characterized by two quantum numbers: the total spin $S_{z}$ and the total angular momentum $M_{z}$. The persistent current is obtained for two interacting rings without tunneling between them, containing each three electrons, at different interring distances. As shown in Fig. 1(a), the current is an oscillation function of the magnetic field with semilinear segments. The different segments appear because $M_{z}$ increases with increasing magnetic field leading to a GS configuration (or phase) transition as indicated in the figure. At small magnetic fields, the GS phase of a three-electron $\mathrm{QR}$ is $\left(S_{z}, M_{z}\right)=(1 / 2,1)$. With increasing magnetic field, the total angular momentum increases as $M_{z}=1,2,4$, and 5, but the total spin remains $S_{z}=1 / 2$. We observe that the position of the jumps in the current is not a monotonous function of $d$. Figure 1(b) presents a phase diagram of the ground states of the QRs in the $B$ vs $d$ plane. The horizontal dotted lines indicate the GS transitions of a single ring. The GS depends on the interring distance $d$. Moreover this dependence is stronger when $d$ is close to the diameter of the rings $\left(2 r_{0}\right)$. It results mainly from the interring Coulomb energy which is different for the different GS configurations (different $M_{z}$ ) as shown in the inset of Fig. 1(b). The energy difference $\Delta E^{\text {inter }}$ of the interring coulomb energy $E_{S_{2}, M_{z}}^{\mathrm{inter}}$ between the different configurations $\left(S_{z}, M_{z}\right)$ and $\left(S_{z}^{\prime}, M_{z}^{\prime}\right)$ is plotted as a function of $d ; \Delta E^{\text {inter }}$ $=E_{S_{2}, M_{z}}^{\text {inter }}-E_{S^{\prime}, M^{\prime}}^{\text {inter }}$. The three curves are $E_{1 / 2,2}^{\text {inter }}-E_{1 / 2,1}^{\text {inter }}$ at $B$ $=0.85 \mathrm{~T}, E_{1 / 2,4}^{z_{i}}-E_{1 / 2,2}^{\text {inter }}$ at $B=1.4 \mathrm{~T}$, and $E_{1 / 2,5}^{\text {inter }}-E_{1 / 2,4}^{\text {inter }}$ at $B$ $=2.0 \mathrm{~T}$. The energy difference is zero for $d \approx 2 r_{0}$. In comparison with the single ring, the phase transition occurs at lower (larger) magnetic fields when $d<2 r_{0}\left(d>2 r_{0}\right)$. As a consequence, we found the remarkable behavior that the interring Coulomb interaction affects significantly the persistent current. Figure 1(c) shows that at $B=0.7$ and $1.2 \mathrm{~T}$, where the GS configurations are the same for all $d$, the persistent current decreases with increasing $d$ until minimum and then increases slowly to its limit value of an isolated ring. However, variation in the interring distance at fixed magnetic field can also induce the GS phase transition and, consequently, a jump in the persistent current. This is the case of $B=0.85 \mathrm{~T}$ in Fig. 1(c) where the GS configuration changes from $(1 / 2,2)$ to $(1 / 2,1)$ at $d=1.2 r_{0}$ leading to an abrupt jump of the persistent current from -4 to $22 \mathrm{nA}$.

The CQRs form an artificial molecule when interring quantum tunneling is permitted. Figure 2(a) shows the persistent current as a function of magnetic field in such an artificial molecule with a total of three electrons in the strong
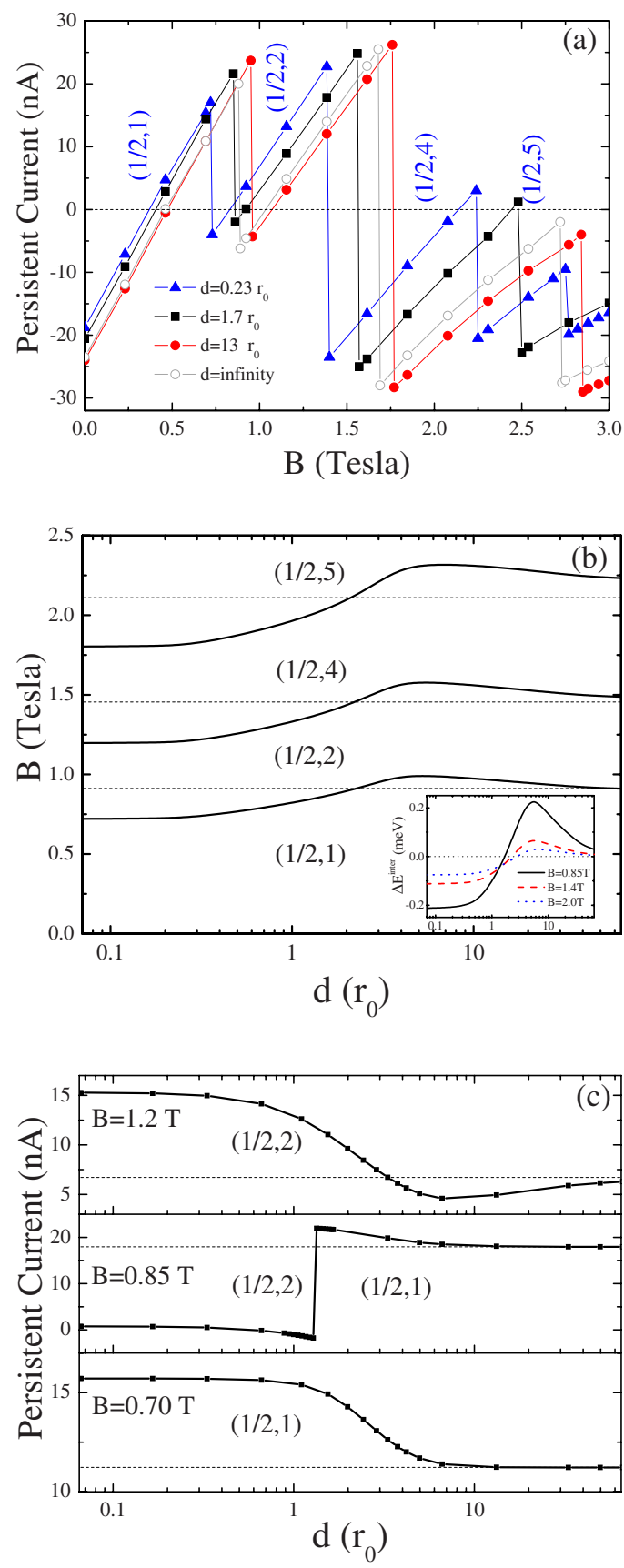

FIG. 1. (Color online) (a) The persistent current as a function of magnetic field at different $d$ in two interacting QRs containing each three electrons without interring tunneling. (b) The phase diagram in the $B-d$ plane. The dashed horizontal lines indicate the transitions in a single ring. Each phase is indicated by two quantum numbers $\left(S_{z}, M_{z}\right)$. The inset shows the energy difference $\Delta E^{\text {inter }}$ of the interring Coulomb energy between the different configurations at a fixed magnetic field. (c) Variation in the persistent current in the rings as a function of $d$ at $B=0.7,0.85$, and $1.2 \mathrm{~T}$. The horizontal lines are the current for the single ring case.

tunneling regime for $d=0.1 r_{0}$. In this regime, the CQRs behave as a single one because only the bonding states are occupied. This is evident by the isospin quantum number $I_{z}$ which is always $3 / 2$. The spin and the angular momentum $\left(S_{z}, M_{z}\right)$ follows the same sequence as those in Fig. 1(a). 


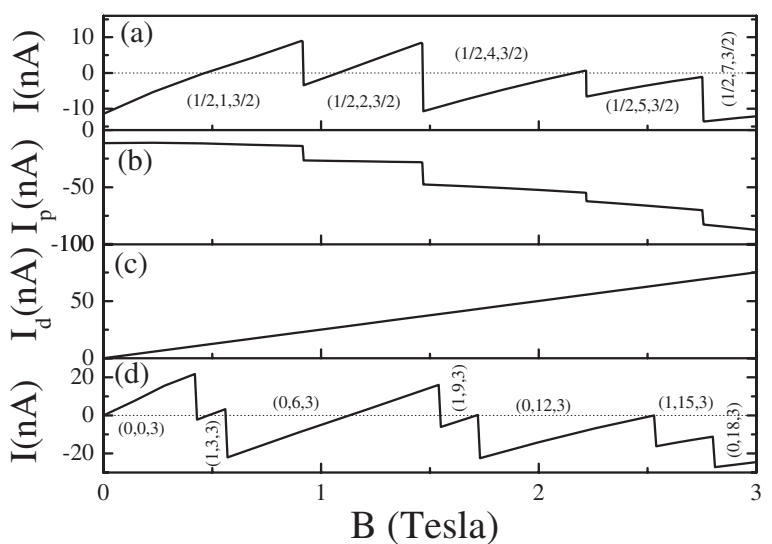

FIG. 2. (a) The total persistent, (b) the paramagnetic, and (c) the diamagnetic currents as a function of magnetic field in the CQRs of $d=0.1 r_{0}$ and with $N=3$ electrons. (d) The same as (a) but now for $N=6$. The different phases are indicated by $\left(S_{z}, M_{z}, I_{z}\right)$.

Figures 2(b) and 2(c) give the corresponding paramagnetic and diamagnetic currents, respectively. The paramagnetic current jumps with the GS phase change while the diamagnetic current is a linear function of the magnetic field. The total momentum increases with increasing magnetic field with jumps $\Delta M_{z}=(N \pm 1) / 2$. But the total spin and isospin remain $S_{z}=1 / 2$ and $I_{z}=N / 2$.

Figure 2(d) presents the persistent current in the CQRs of six electrons at $d=0.1 r_{0}$ which is in the strong tunneling regime where $I_{z}=N / 2=3$. When the electron number is even $(N=6)$, with increasing magnetic field, the total angular momentum always increase by $\Delta M_{z}=N / 2$ when the system transits from one phase to the next as indicated in the figure. The total spin oscillates between 0 and 1 . The most stable states correspond to the closed-shell configurations $\left(S_{z}=0\right)$. When the total spin is nonzero $\left(S_{z}=1\right)$, the ground state is less stable because it corresponds to an open-shell system.

In the cases of a single ring and the strongly coupled double rings, new GS phases appear with increasing magnetic field due mainly to the increase in the total angular momentum. However, with increasing interring distance, when the tunneling splitting energy between the bonding and antibonding levels reduces to compete with the interring exchange-correlation energy, the molecular phases can be complex and so the persistent current.

In order to understand better the persistent current in the CQRs in the intermediate and weak tunneling regime, we plotted in Fig. 3 the phase diagram of the CQRs of six electrons (on the average three electrons per ring) in the $B-d$ plane. The phase diagram of the corresponding two ring case without tunneling was presented in Fig. 1(b). It is seen that for small $d$, the coupled rings behave similarly to a single one because of the strong tunneling (large $\Delta_{\mathrm{SAS}}$ ). All the electrons are in the bonding states in this case. With increasing $d$, the coupled rings are in the so-called molecular phase. For $0.15 r_{0} \leq d \leq 0.4 r_{0}$, competition between the tunneling energy $\Delta_{\mathrm{SAS}}$ and the electron exchange energy leads to a quite complex phase diagram. The electrons occupy the antibonding state but most of the electrons are in the bonding state $\left(I_{z}=1\right.$ or 2$)$. At large $d, \Delta_{\text {SAS }}$ approaches zero, the same

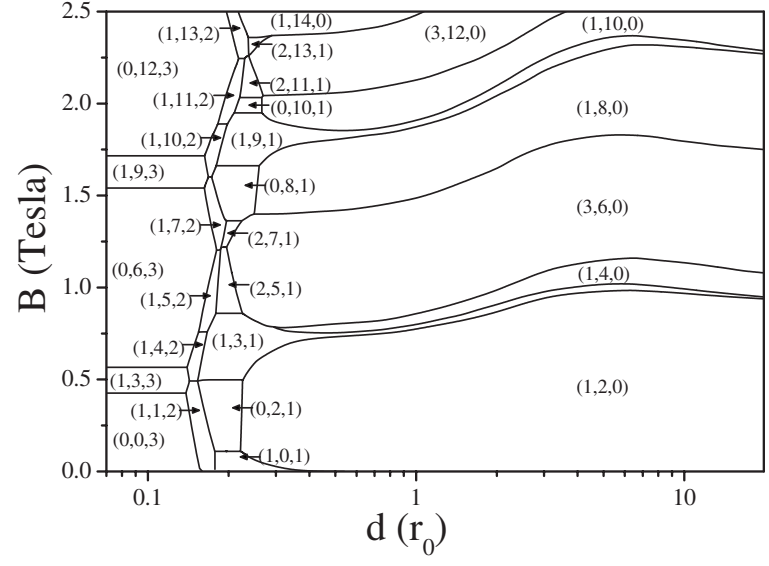

FIG. 3. The phase diagrams of the ground-state configurations of the CQRs of $N=6$ electrons. The three quantum numbers $\left(S_{z}, M_{z}, I_{z}\right)$ indicate the ground-state configuration.

number of electrons are in the bonding and antibonding states so $I_{z}=0$. In this case, the interring direct Coulomb interaction and the exchange energy dominate the GS phases. In comparison with Fig. 1(b), the interring tunneling alters the phase diagram dramatically. For instance, in the phase $(3,6,0)$, three electrons are in the bonding and three electrons in the antibonding state. All these six electrons have parallel spin. In a real sample, the tunneling at large interring separation can be controlled by a weak external electric field in the $z$ direction.

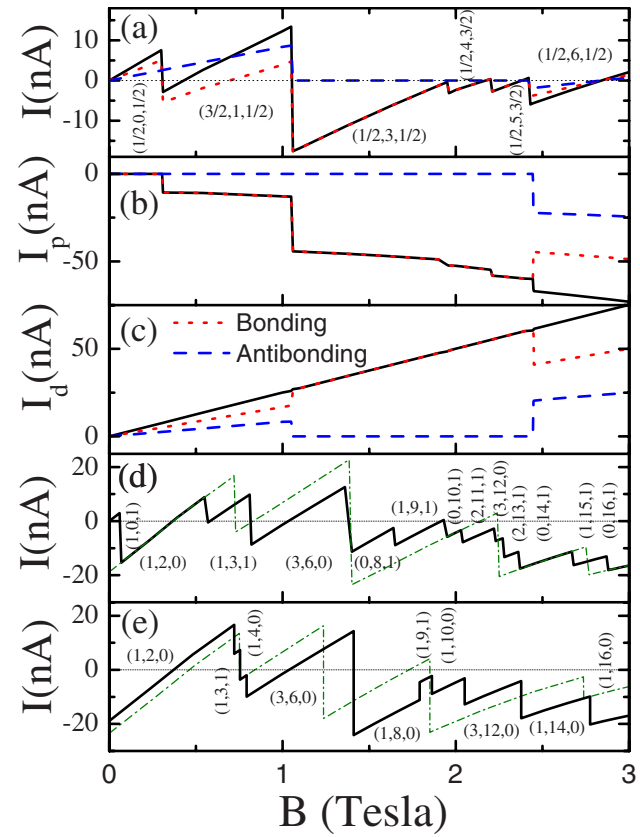

FIG. 4. (Color online) (a) The persistent, (b) the paramagnetic, and (c) the diamagnetic currents in the CQRs with $d=0.23 r_{0}$ and $N=3$ electrons. The dotted (red) and dashed (blue) curves are currents carried by the bonding and antibonding states, respectively. The persistent current in the CQRs of $N=6$ electrons at (d) $d$ $=0.23 r_{0}$ and (e) $0.40 r_{0}$. The thin dash-dotted curves are the current in the corresponding coupled rings without tunneling. Each GS phase is indicated by $\left(S_{z}, M_{z}, I_{z}\right)$. 
In the intermediate tunneling regime (e.g., $d=0.23 r_{0}$ ), the CQRs are in the molecular phase. Figure 4(a) shows the persistent currents as a function of the magnetic field in the CQRs of three electrons. In this regime both the bonding and antibonding states are occupied as indicated by the value of $I_{z}$. Therefore, more combinations of angular momenta become possible as a function of the magnetic field leading to more oscillations in the persistent current. At large magnetic fields, the rapid variation in the angular momentum with increasing magnetic field leads to a suppression of the amplitude of the persistent current. The dotted (red) and dashed (blue) curves indicate the currents carried by the electrons in the bonding and antibonding states, respectively. The black curve represents the total current, which is a sum of the currents due to the bonding and antibonding states. Figures 4(b) and 4(c) show the corresponding paramagnetic and diamagnetic currents, respectively. In Fig. 4(b), we see that the paramagnetic current due to the antibonding state is zero until $B=2.35$ T where the $(1 / 2,6,1 / 2)$ state appears. An increase in the current due to antibonding states is always partially compensated by a decrease in the current due to the bonding states. Such a compensation is complete for the diamagnetic current as shown in Fig. 4(c).

Figures 4(d) and 4(e) show the persistent current as a function of the magnetic field in the CQRs of six electrons at $d=0.23 r_{0}$ and $0.40 r_{0}$, respectively. The GS phase diagram of these CQRs is given in Fig. 3. The dotted-dash (green) curves in Figs. 4(d) and 4(e) give the current in the corresponding rings without tunneling (three electrons in each ring). Clearly the tunneling leads to different ground-state phases and consequently different persistent current. We no- tice that in the CQRs of $d=0.23 r_{0}$, the persistent current is zero at $B=0$ [see Fig. 4(d)] when there is tunneling between the two rings. But this current is about $-20 \mathrm{nA}$ when the tunneling is absent. We know that a vertical gate potential can control tunneling in such a structure. It means that an external gate can switch on/off the persistent current (and the magnetization) of these coupled rings even in the absence of a magnetic field.

\section{CONCLUSIONS}

In summary, the only external parameter which controls the persistent current in a single $\mathrm{QR}$ is the external magnetic field. In this calculation we show that in coupled quantum rings, the persistent currents in the CQRs can be controlled by the interring coupling. This interring coupling consists of the interring Coulomb interaction and the interring quantum tunneling. The former depends not only on the interring distance but also on the electronic configuration of the QRs which can be modulated by an external magnetic field. The latter depends on the interring tunneling energy, the interring electron exchange effects, as well as the external magnetic field and can also be controlled by a vertical gate.

\section{ACKNOWLEDGMENTS}

This work was supported by FAPESP and CNPq (Brazil) and by the Flemish Science Foundation (Contract No. FWOVl) and the Belgium Science Policy (IAP), Belgian State Policy.

\footnotetext{
*hai@if.sc.usp.br

${ }^{1} \mathrm{~J}$. Hamilton, Aharonov-Bohm and Other Cyclic Phenomena (Springer-Verlag, Berlin, 1997).

${ }^{2}$ N. A. J. M. Kleemans et al., Phys. Rev. Lett. 99, 146808 (2007).

${ }^{3}$ D. Granados, J. M. Garcia, T. Ben, and S. I. Molina, Appl. Phys. Lett. 86, 071918 (2005); E. Marega, Z. Y. Abuwaar, and G. Salamo (unpublished).

${ }^{4}$ L. K. Castelano, G.-Q. Hai, B. Partoens, and F. M. Peeters, Phys. Rev. B 74, 045313 (2006), references therein; G. Piacente and G.-Q. Hai, J. Appl. Phys. 101, 124308 (2007).

${ }^{5}$ F. Malet, M. Barranco, E. Lipparini, R. Mayol, M. Pi, J. I. Climente, and J. Planelles, Phys. Rev. B 73, 245324 (2006); M.
}

Royo, F. Malet, M. Barranco, M. Pi, and J. Planelles, arXiv:0809.3103, Phys. Rev. B (to be published).

${ }^{6}$ B. Szafran, Phys. Rev. B 77, 235314 (2008); 77, 205313 (2008).

${ }^{7}$ Y. Z. He and C. G. Bao, J. Phys.: Condens. Matter 20, 055214 (2008).

${ }^{8}$ G. Vignale and M. Rasolt, Phys. Rev. Lett. 59, 2360 (1987); Phys. Rev. B 37, 10685 (1988).

${ }^{9}$ S. Viefers, P. S. Deo, S. M. Reimann, M. Manninen, and M. Koskinen, Phys. Rev. B 62, 10668 (2000).

${ }^{10}$ B. Partoens and F. M. Peeters, Europhys. Lett. 56, 86 (2001); Phys. Rev. Lett. 84, 4433 (2000). 